\section{La Révolution française}

Cahiers de l'Institut d'histoire de la Révolution française

$21 \mid 2021$

Les Archives parlementaires entre papier et toile : exploitation d'une source [numéro spécial]

\title{
Accuser les conventionnels en l'an III. Les débats parlementaires à la lumière des archives du Comité de Législation
}

Jean d'Andlau

\section{OpenEdition}

\section{Journals}

Édition électronique

URL : https://journals.openedition.org/Irf/5629

DOI : $10.4000 /$ Irf.5629

ISSN : 2105-2557

Éditeur

IHMC - Institut d'histoire moderne et contemporaine (UMR 8066)

Référence électronique

Jean d'Andlau, « Accuser les conventionnels en l'an III. Les débats parlementaires à la lumière des archives du Comité de Législation », La Révolution française [En ligne], 21 | 2021, mis en ligne le 25 octobre 2021, consulté le 27 octobre 2021. URL : http://journals.openedition.org//rf/5629 ; DOI : https://doi.org/10.4000//rf.5629

Ce document a été généré automatiquement le 27 octobre 2021

(C) La Révolution française 


\section{Accuser les conventionnels en l'an III. Les débats parlementaires à la lumière des archives du Comité de Législation}

Jean d'Andlau

1 Dans l'éditorial du dernier numéro de son éphémère journal Le Peuple constituant, Félicité de Lamennais évoque, le 11 juillet 1848, au sujet des ouvriers décimés par la répression, « des transportations en masse, des proscriptions telles que 95 n'en fournit pas d'exemples ${ }^{1} »$. Cette légende noire de 1795 , si longtemps entretenue dans l'historiographie de la Révolution ${ }^{2}$, trouve en partie ses origines dans le rôle qu'aurait joué le Comité de Législation au cours de l'an III. Anticipant le propos de Lamennais, le conventionnel Marc Antoine Baudot évoque ainsi, dans ses mémoires, un comité « qui envoyait les députés républicains à la mort $^{3} »$. L'étude renouvelée des débats parlementaires, associée à l'examen des archives du comité, permet de réinterroger le rôle d'un comité longtemps dépeint comme triste artisan du "miroir sans magie " thermidorien ${ }^{4}$, alors que les travaux de Mette Harder ${ }^{5}$, de Michel Biard ${ }^{6}$, d'Howard Brown $^{7}$ et, enfin, l'ouvrage collectif Visages de la Terreur ${ }^{8}$ nous encouragent à déconstruire une opposition binaire et manichéenne entre l'an II et l'an III, à mettre en lumière les complexités d'une période thermidorienne passée à la postérité au travers de topoï avant tout destinés à l'auto-absolution de la Convention et de ses membres9

En effet, comme l'écrivait Françoise Brunel, l'histoire de la mise en accusation des conventionnels en l'an III est aussi celle du «moment thermidorien $»^{10}$. Dans un premier temps, c'est ainsi l'unité qui semble primer. Le 13 thermidor (31 juillet), Legendre demande que soit restauré une forme d'immunité parlementaire ${ }^{11}$. Au cours des débats, afin d'escamoter la question des responsabilités collectives, Bentabole justifie les précédentes mises en accusation de législateurs en dénonçant des décrets "surpris à la Convention ${ }^{12}$ ». Passé un moment de relatif apaisement, les luttes politiques et les insurrections en province remettent dès l'automne au centre des 


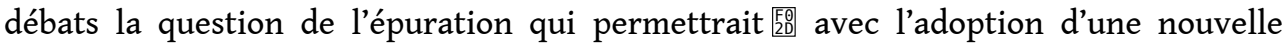
Constitution 医 de "clore la Révolution", de réaliser enfin le projet politique thermidorien : tourner la page de la Terreur et fonder la République.

\section{Les comités face aux dénonciations}

3 C'est la vindicte de Lecointre, dénonciateur acharné, qui ouvre la porte à une mise en cause d'autres figures de l'an II alors que se délite progressivement l'unité de façade forgée autour des événements de thermidor ${ }^{13}$. Dans le même temps, la difficile réforme du tribunal révolutionnaire ${ }^{14}$ et les interrogations sur la légitimé des représentants en mission ${ }^{15}$ montrent bien toute la difficulté qu'ont les thermidoriens à rejeter en bloc l'héritage de l'an II, à faire porter à Robespierre et à quelques autres les habits de croque-mitaine ${ }^{16}$. Ces mises en accusation reflètent une évolution vers la droite de l'Assemblée, qui ouvre la voie au procès des «quatre grands coupables ${ }^{17}$, Barère, Billaud, Collot et Vadier, mais aussi à la mise en cause d'autres figures emblématiques de l'an II, comme Vouland, Amar et David. Inclure dans les boucs émissaires certains représentants en mission ainsi que d'anciens membres des comités de gouvernement permettait d'exonérer le reste de l'Assemblée d'une quelconque participation au système de la terreur.

4 Craignant cependant d'ouvrir la porte à un flot de dénonciations qui les emporte tous « jusqu'à la sonnette du président ", pour reprendre la formule prêtée à Carrier ${ }^{18}$, la Convention confie à ses trois comités l'élaboration d'une procédure qui permettent de garantir la représentation nationale dans les accusations portées contre ses membres. Il s'agit d'éviter toute dérive, de ne pas donner à ces procès l'apparence d'un second 31 mai. Le résultat de ce travail est présenté par Merlin de Douai au cours de la journée du 8 brumaire an III (29 octobre 1794) ${ }^{19}$.

5 Un certain nombre d'étapes précises se suivent. Dans un premier temps, toute dénonciation contre un représentant du peuple doit être renvoyée aux trois comités réunis. Les comités étudient ensuite ces dénonciations et décident s'ils doivent en rendre compte à la Convention. Si les trois comités estiment qu'une dénonciation mérite considération, ils soumettent alors une déclaration commune à la Convention et présentent les faits. Après ce rapport, une commission de vingt-et-un députés est désignée par le sort et rédige un rapport sur les faits et les éléments de preuve produits. Ce rapport terminé, le décret d'accusation ne peut être décidé qu'après un appel nominal. Enfin, il est décidé que le prévenu puisse faire imprimer et distribuer aux membres de la Convention toutes les pièces qu'il juge utiles à sa défense et qu'il soit par ailleurs présent lors de la discussion du rapport et puisse être entendu sur les faits.

6 Ce décret est adopté sans aucune opposition. Seules quelques propositions sont formulées pour le compléter, propositions rapidement envoyées mûrir dans les comités, pour ne plus jamais revenir en séance. Or cette procédure ne va pas tenir bien longtemps. L'aggravation des tensions, puis l'insurrection du 12 germinal ( $1^{\text {er }}$ avril 1795) vont conduire à l'adoption de mesures qui court-circuitent largement les engagements pris en brumaire.

7 Le 21 floréal an III (10 mai 1795), la Convention débat de la réorganisation du gouvernement et de la répartition des pouvoirs entre les comités. Au cours des échanges, Fréron dénonce avec virulence les deux grands comités : «en dernière analyse, je ne vois, dans toutes ces attributions données aux deux comités de 
gouvernement, que les ressorts d'une tyrannie plus vaste, mieux combinée, plus épouvantable même que celle de Robespierre ${ }^{20} »$. C'est à ce moment que son collègue Louvet demande et obtient que l'examen de la conduite des Conventionnels soit retiré aux comités. L'Assemblée décrète alors « qu'en aucun cas les Comités de salut public et de sûreté générale ne seront chargés de l'examen de la conduite des représentants du peuple ${ }^{21} »$. Le Comité de Législation reste alors seul chargé de l'examen des dénonciations, ainsi que de présenter à l'Assemblée un rapport s'il le juge nécessaire. Soit qu'il s'agisse d'éloigner le spectre d'un jugement politique par les comités de gouvernement, soit que l'objectif ait été d'accélérer les procédures alors que les émeutes populaires de germinal et de prairial ébranlent les fondements de la république thermidorienne, l'Assemblée décide de confier aux membres d'un unique comité l'écrasante responsabilité de désigner ceux de leurs collègues dont la conduite justifierait l'arrestation et la mise en accusation. Première entorse à la procédure.

8 L'essentiel des dénonciations parvient dès lors au comité, dont les nouvelles attributions reflètent bien la confiance que lui accorde la Convention, tout comme la place prise par les hommes de la Plaine au cours de l'été 1794. Dans ce délicat exercice d'équilibre qui s'annonce, on est loin des palinodies dénoncées par Albert Mathiez et George Lefebvre qui, hommes de leur temps, voyaient dans ces légistes des "girouettes ${ }^{22}$ " destinées à dévoyer la Révolution populaire au profit d'intérêts bourgeois $^{23}$. Le Comité de Législation s'affirme en effet comme une véritable antichambre de la justice thermidorienne. Ses membres agissent avec grande prudence, en suivant scrupuleusement la procédure établie en brumaire ${ }^{24}$. Dans un premier temps, les commissaires constituent les dossiers et demandent des pièces supplémentaires aux comités de gouvernement ou aux administrations ${ }^{25}$. Vient ensuite le temps de l'analyse individuelle des dossiers avec dépositions des témoins et des accusés. Au terme de ces enquêtes, le comité décide s'il convient ou non de présenter le cas à leurs collègues de la Convention. On trouve d'ailleurs dans les archives conservées une liste de 98 députés, répartis en trois colonnes intitulées "noms des représentants en faveur desquels il a été délibéré n'y avoir lieu à plus ample examen", "noms de ceux auxquels il a été délibéré d'écrire pour les entendre et en faveur desquels il a été arrêté de passer à l'ordre du jour", "noms de ceux qui doivent être portés à la Convention nationale ${ }^{26}$.

9 Dans ses mémoires, Cambacérès, longtemps inamovible président du Comité de Législation, revient sur ce travail lourd de responsabilités. Le Montpelliérain assure que son comité « rejeta les dénonciations vagues, repoussa celles qui étaient fausses et ne proposa point de sévir contre des fautes que l'enthousiasme de la liberté pouvait faire pardonner ». Et d'ajouter : «si l'avis du comité avait été suivi, il n'aurait été pris aucune mesure de rigueur ${ }^{27} »$.

10 Prudent et scrupuleux dans son travail, le comité, qui devait rester maitre de son calendrier, est cependant plusieurs fois pressé, contraint de présenter ses rapports sans tarder. Au cours de la séance du 5 prairial an III (24 mai 1795), à la demande du réacteur Dumont, ses membres sont enjoints de présenter sans tarder un rapport sur les représentants accusés "d'avoir fait couler le sang innocent, ou commis des dilapidations dans les missions qui leur ont été confiées». Comme le souligne Sergio Luzzatto, avec ce décret, les conventionnels, qui avaient pour beaucoup dénoncé les mesures d'exception de l'an II largement déconstruites depuis thermidor, choisissent donc la vieille tactique de la procédure exceptionnelle, ponctuelle et improvisée plutôt 
que de s'en remettre à l'application de la feuille de route votée le 8 brumaire (29 octobre $)^{28}$.

11 Ce décalage entre la procédure voulue par les conventionnels et sa mise en œuvre en l'an III est particulièrement visible dans les rapports que présente le Comité de Législation lors des séances du 13 prairial ( $1^{\mathrm{er}}$ juin) et du 21 thermidor (8 août) an III. Ces rapports, présentés à la tribune et dont Le Moniteur universel a conservé la trace, permettent de mieux saisir à la fois le travail du Comité de Législation, mais aussi l'évolution de la justice thermidorienne au cours de l'an III.

\section{Le rapport du 13 prairial an III (1 er juin 1795)} dénonciations contre les représentants accusés d'avoir "fait couler le sang innocent $»^{32}$, le comité a considéré comme «sang innocent » la mort de toute personne hors des formes prévues par la loi. Plus encore, Durand-Maillane ajoute que ses collègues et lui-même ont pris l'initiative d'élargir le champ d'accusation. Le rapporteur, canoniste de formation, précise ainsi avoir pris en compte d'autres crimes, à l'image de la "réquisition forcée de filles et de femmes", crime "d'une immoralité inouïe qui sape par sa base un gouvernement fondé principalement sur les bonnes mœurs des citoyens $»^{33}$.

15 S'ensuit, pour chaque député mis en cause, une présentation des pièces justificatives, résumées ou lues in extenso. L'étude de ces pièces permet de montrer que ce rapport du 13 prairial est bien loin de la simple présentation des faits demandée par la Convention. Peu nombreux, souvent brièvement résumés, ces documents ont été plutôt choisis pour cibler les députés mis en cause.

Tableau 1: Liste des députés dont la conduite est examinée au cours des séances du 13 prairial an III et pièces produites par le rapporteur

\begin{tabular}{|l|l|}
\hline Député mis en cause & Pièces produites par le comité \\
\hline $\begin{array}{l}\text { Pierre-Arnaud Dartigoeyte } \\
\text { Député des Landes }\end{array}$ & $\begin{array}{l}\text { Dénonciation du conventionnel Joachim Perez } \\
\text { Procès-verbaux de la société populaire d'Auch }\end{array}$ \\
\hline
\end{tabular}




\begin{tabular}{|c|c|}
\hline $\begin{array}{l}\text { Antoine Louis François } \\
\text { Sergent } \\
\text { Député de la Seine }\end{array}$ & $\begin{array}{l}\text { Déclaration d'un vétéran de la garde assurant l'avoir entendu } \\
\text { prononcer des propos séditieux le } 1^{\mathrm{er}} \text { prairial } \\
\text { Circulaire du comité de surveillance de la commune de Paris, signée } \\
\text { par Sergent, appelant au massacre des prisonniers en septembre } 1792 \\
\text { Dénonciations pour vol et enrichissement personnel }\end{array}$ \\
\hline $\begin{array}{l}\text { Nicolas Maure } \\
\text { Député de l'Yonne }\end{array}$ & Une accusation verbale d'un de ses collègues députés \\
\hline $\begin{array}{l}\text { François René Mallarmé } \\
\text { Député de la Meurthe }\end{array}$ & Synthèse de dénonciations individuelles \\
\hline $\begin{array}{l}\text { Claude Javogues } \\
\text { Député de Rhône-et-Loire }\end{array}$ & Lettre de dénonciation d'une commune du Puy-de-Dôme \\
\hline $\begin{array}{l}\text { Élie Lacoste et Marc } \\
\text { Antoine Baudot } \\
\text { Député de la Dordogne et } \\
\text { de la Saône-et-Loire }\end{array}$ & Lettres de dénonciation remises au Comité de Législation \\
\hline $\begin{array}{l}\text { Jean-Baptiste-Benoît } \\
\text { Monestier } \\
\text { Député du Puy-de-Dôme }\end{array}$ & Lettres de dénonciation remises au Comité de Législation \\
\hline $\begin{array}{l}\text { Sylvain-Phalier Lejeune } \\
\text { Député de l'Indre }\end{array}$ & Accusation des administrateurs du district de Besançon \\
\hline $\begin{array}{l}\text { Pierre Alard } \\
\text { Député de la Haute- } \\
\text { Garonne }\end{array}$ & Lettres de dénonciation remises au Comité de Législation \\
\hline
\end{tabular}

À l'étude de ces pièces, un fossé apparaît entre la prudence affichée par DurandMaillane et les dénonciations sinon à l'emporte-pièce, du moins légères, présentées par ce rapport. Dartigoeyte ou Lejeune sont ainsi décrétés d'arrestation alors qu'une seule dénonciation est retenue par le comité. Sergent est décrété d'arrestation pour des faits connus de tous, assez anciens, et qu'il a eu plusieurs fois l'occasion de démentir. Dugué d'Assé, qui prend la relève de Durand-Maillane, ne présente dans les cas de Mallarmé ou des missionnaires Jean-Baptiste Lacoste et Baudot, qu'une synthèse de lettres remises au comité. Les faits sont certes nombreux, mais ressemblent davantage à une dénonciation reprenant les poncifs du conformisme politique de l'an III $^{34}$. Mallarmé est ainsi accusé de "n'avoir jamais rien payé » au cours de ses déplacements ${ }^{35}$. On peut difficilement imaginer qu'il soit le seul député en mission à avoir procédé de la sorte.

Parfois, les accusations n'arrivent même pas à convaincre. L'Assemblée renvoie ainsi à son comité le cas de Maure, qu'elle juge insuffisamment étayé. On est loin des preuves irréfutables, du "tableau fidèle", que Durand-Maillane s'était engagé à présenter. Même Legendre, pourtant ardent réacteur, est obligé de reconnaître qu'il faut que « la Convention soit sévère, mais il faut également qu'elle soit juste » et ajoute à propos de Maure qu'« il n'y a ici qu'une déposition, et si, sur l'attestation d'un seul homme, la 
Convention se permettait de faire arrêter ses membres, il en résulterait que très souvent la haine et les vengeances particulières détermineraient de pareilles mesures $^{36}$ ».

19 Par ailleurs, la lecture des pièces est systématiquement suivie de vifs débats, parfois pathétiques, à l'exemple des attaques de Dentzel, député du Bas-Rhin, contre Lacoste, le premier accusant l'ancien représentant en mission de porter une chemise qu'il lui aurait dérobée lors de sa mission dans son département d'origine ${ }^{37}$. Ce sont davantage ces accusations formulées en séance qui emportent la décision finale et les pièces proposées par le comité ne sont que rarement commentées. En prairial, elles ne semblent donner qu'un " voile de légitimité » aux attaques ciblées, à des procès " jugés mais non plaidés ", pour reprendre une expression de Cambacérès ${ }^{38}$.

20 À la fin de la journée, neuf députés, tous montagnards, sont décrétés d'arrestation. Seuls six étaient présents et ont pris la parole pour se défendre. On est bien loin de la prudence affichée en brumaire. À ce stade, et comme l'a très bien montré Françoise Brunel, l'objectif des mises en accusation est autant de juger les crimes de l'an II que d'épurer la Convention finissante, d'éloigner les députés vus comme les derniers ferments de la discorde. Fréron ne dit pas autre chose lorsqu'il s'exprime au sujet de certains députés mis en cause: «ils ont cru noyer dans le sang la révolution du 9 thermidor ; mais non, elle va recevoir son complément ${ }^{39} »$. Il s'agit bien de purger une deuxième et dernière fois la Convention.

\section{Le rapport des 21 et 22 thermidor an III (8 et 9 août 1795)}

21 S'il présente de nombreux points communs avec celui que nous avons précédemment évoqué, ce rapport reflète clairement un changement dans le climat politique thermidorien. Ce sont, certes, une fois encore, les députés montagnards, au nombre de onze, qui sont visés, mais l'heure n'est plus à la continuation des purges de thermidor. Les faits sont davantage étayés et les accusés invités à se défendre.

Ici, le travail du Comité de Législation est présenté par Girod-Pouzol, Bézard et Genissieu. Girod-Pouzol, comme Durand Maillane avant lui, commence son rapport en rappelant qu'en brumaire, il n'a pas été demandé à son comité de prononcer des "mesures de rigueur", mais bien de faire connaître les dénonciations qui visent plusieurs représentants du peuple. Il assure ensuite à ses collègues que le comité a procédé à un véritable travail d'enquête pour vérifier les faits et écarter les dénonciations calomnieuses et qu'il a "voué au mépris » les dénonciations envoyées sous le « voile de l'anonyme $»^{40}$. Il confie ensuite la parole à son collègue Bézard, chargé de présenter les pièces contre les représentants du peuple.

L'étude de la présentation de ce second rapport permet de souligner que, lorsque le contexte politique le permet, les conventionnels savent renouer opportunément avec les procédures qu'ils avaient mises en place.

24 Tableau 2 : Liste des députés dont la conduite est examinée au cours des séances des 21 et 22 thermidor an III et pièces produites par le rapporteur

\begin{tabular}{|l|l|}
\hline Député & Pièces produites par le comité
\end{tabular}




\begin{tabular}{|c|c|}
\hline $\begin{array}{l}\text { Joseph François Laignelot et } \\
\text { Joseph Lequinio } \\
\text { Députés de la Seine et du } \\
\text { Morbihan }\end{array}$ & $\begin{array}{l}\text { Dénonciation de la commune de Rochefort } \\
\text { Une lettre de dénonciation accusant Lequinio de vol de numéraire } \\
\text { Mémoire justificatif de Lequinio }\end{array}$ \\
\hline $\begin{array}{l}\text { Antoine-Joseph Lanot } \\
\text { Député de la Corrèze }\end{array}$ & $\begin{array}{l}\text { Deux dénonciations de citoyens de la commune de Brive } \\
\text { Défense de Lanot auprès du Comité de Législation }\end{array}$ \\
\hline $\begin{array}{l}\text { Jean-Alban Lefiot } \\
\text { Député de la Nièvre }\end{array}$ & Un arrêté compromettant signé de sa main \\
\hline $\begin{array}{l}\text { André Dupin } \\
\text { Député de l'Aisne }\end{array}$ & Plusieurs dénonciations relatives à l'affaire des fermiers généraux \\
\hline $\begin{array}{l}\text { Jean-Baptiste Bô } \\
\text { Député de l'Aveyron }\end{array}$ & $\begin{array}{l}\text { Dénonciation de la commune de Reims } \\
\text { Dénonciations de citoyens du Lot } \\
\text { Mémoire justificatif de Bô }\end{array}$ \\
\hline $\begin{array}{l}\text { Pierre-François Piorry } \\
\text { Député de la Vienne }\end{array}$ & Une lettre de Piorry à la Société populaire de Poitiers \\
\hline $\begin{array}{l}\text { Jean-Baptiste Massieu } \\
\text { Député de l'Oise }\end{array}$ & $\begin{array}{l}\text { Dénonciations des autorités constituées du département des } \\
\text { Ardennes et des citoyens de Sedan }\end{array}$ \\
\hline $\begin{array}{l}\text { Guillaume Chaudron- } \\
\text { Rousseau } \\
\text { Député de la Haute-Marne }\end{array}$ & Lettre de dénonciation de citoyens du département de l'Aude \\
\hline $\begin{array}{l}\text { Jacques Léonard Laplanche } \\
\text { Député de la Nièvre }\end{array}$ & $\begin{array}{l}\text { Dénonciation des autorités constituées du département de la } \\
\text { Nièvre et de la commune de Nevers }\end{array}$ \\
\hline $\begin{array}{l}\text { Joseph Fouché } \\
\text { Député de la Loire-Inférieure }\end{array}$ & $\begin{array}{l}\text { Procès-verbaux des séances publiques de différentes } \\
\text { administrations et des propositions faites par Fouché } \\
\text { Lettres de Fouché aux administrateurs de la Nièvre } \\
\text { Mémoire de défense adressé par Fouché }\end{array}$ \\
\hline
\end{tabular}

Ce deuxième rapport est bien différent du travail présenté en prairial.

On n'y trouve pas de synthèse, d'amas de faits épars comme ce fut le cas quelques semaines plus tôt. Au contraire, les allégations sont étayées avec prudence, les droits des mis en cause sont davantage respectés. Ainsi, Piorry est certes décrété d'arrestation sur une lettre qu'il a écrite et dans laquelle il appelle les «vigoureux sans-culottes » à " tout briser, tout renverser, tout incendier, tout déporter, tout renfermer, tout guillotiner, tour régénérer ", mais il a l'occasion de présenter sa défense et reconnaît avoir écrit la lettre ${ }^{41}$.

Dans le cas de Lefiot, Bézard souligne que les membres du comité ont jugé nécessaire de soumettre à l'Assemblée un arrêté envoyant au tribunal révolutionnaire quatre signataires d'une lettre des citoyens de Montargis exprimant le 29 juin 1792 leur 
soutien au roi après la journée révolutionnaire du 20 juin $^{42}$. Il insiste cependant sur le fait qu'il n'est parvenu au comité aucune autre dénonciation à son sujet ${ }^{43}$. De même, au sujet de Bô, Genissieu annonce que le comité n'a reçu certaines pièces que la veille et qu'il n'a donc pu ni les examiner, ni entendre les députés mis en cause. Néanmoins, avec l'approbation de ses collègues, il quitte la séance pour chercher les documents en question.

La constitution des dossiers reflète aussi davantage l'exigence d'un travail commun, d'une étude de longue haleine et donc un processus plus conforme au texte de brumaire. On trouve ainsi dans les archives du Comité de Législation des nombreux échanges entre les commissaires et les autres comités de gouvernement ${ }^{44}$. Le 13 prairial an III ( $1^{\text {er }}$ juin 1795), le Comité de sûreté générale écrit ainsi au Comité de Législation, pour lui transmettre diverses lettres au sujet du représentant du peuple Massieu, et précise : «le comité vous invite à en tirer tel parti que vous jugerez convenable ». À quoi il adjoint, le 16 prairial (3 juin), trois pièces contre Massieu adressées par le procureur général syndic du département des Ardennes. «Elles pourront contribuer à fixer votre opinion sur le compte de ce député ", écrit le Comité de sûreté générale ${ }^{45}$.

Dans le cas de Massieu, Genissieu semble même endosser le costume de l'avocat lorsqu'il précise ${ }_{20}$ au sujet des méfaits qui auraient été commis lors d'une mission

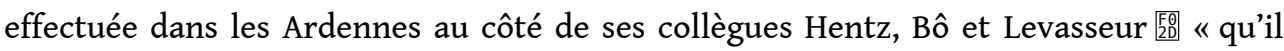
était possible que ses trois autres collègues en aient été les principaux auteurs », et d'ajouter que le comité n'a reçu qu'une seule dénonciation qui le vise explicitement ${ }^{46}$. Visiblement plus attaché aux droits de la défense et aux exigences du décret de brumaire, Genissieu souligne, dans la présentation des faits allégués contre ChaudronRousseau : "Je vous observe maintenant que Chaudron-Rousseau n'étant point encore de retour de sa dernière mission, nous n'avons pu lui communiquer ces dénonciations ni recevoir sa défense ${ }^{47}$.»Et de rajouter, quelque temps plus tard, au sujet de Noël Pointe, attaqué en séance, que si les documents que le comité a en sa possession laissent penser qu'un rapport sur le député est nécessaire, ce rapport ne peut se faire qu'à une séance ultérieure, car le comité n'a pas encore pu entendre l'accusé sur les dénonciations faites contre lui ${ }^{48}$.

Les principaux accusés, lorsqu'ils sont présents, sont d'ailleurs invités à s'exprimer. Ils ont également pu adresser des mémoires au comité, ainsi que des réponses détaillées aux accusations qui les visent ${ }^{49}$. On trouve ainsi dans les archives du comité le mémoire de défense et les attestations adressés par Fouché ${ }^{50}$. Dupin, dénoncé pour son rôle dans le procès des fermiers généraux ${ }^{51}$, produit quant à lui plusieurs lettres pour répondre point par point aux accusations de ses détracteurs. Lequinio, Lanot et Bô envoient également des mémoires justificatifs au comité, lesquels mémoires sont cités ou synthétisés par Bézard lorsqu'il prend la parole auprès de ses collègues.

Cette prise de parole des membres du comité semble ainsi avoir été préparée avec beaucoup plus de soin et de mesure. Les faits présentés sont moins épars, la défense n'est pas négligée, les mis en cause sont invités à se prononcer sur les accusations. Ce changement d'attitude peut en grande partie s'expliquer par le contexte de ce mois d'août 1795 : en effet, l'atmosphère politique a changé depuis les émeutes de germinal et prairial: l'heure n'est plus à la chasse aux jacobins, alors que la reprise de l'insurrection à l'Ouest ou encore l'échec du débarquement de Quiberon viennent réactiver la menace royaliste et rappeler la nécessité de concorde face aux dangers 
extérieurs. Les députés mis en cause trouvent des défenseurs. Tallien ou encore Legendre parlent même en faveur de Fouché !

En somme, ce travail du comité reflète bien la temporalité des luttes politiques qui secouent la Convention en l'an III. Loin des inquiétudes et des désirs de vengeance qui marquent les mois de germinal et de prairial, l'Assemblée cherche cette fois-ci avant tout à assurer le vote de la Constitution, à clore la Révolution. D'ailleurs, aucun de ces députés ne sera jugé. Ce n'était probablement pas l'intention de la Convention. Ces décisions permettent de liquider à peu de frais l'héritage de l'an II et de consolider la place des «hommes nouveaux" aux dépens des derniers Montagnards ${ }^{52}$. En effet, quelques jours plus tard, le 26 octobre 1795 (4 brumaire an IV), la Convention décrète définitivement l'oubli et proclame l'amnistie pour les «faits purement relatifs à la révolution $»^{53}$. La Constitution adoptée, il s'agit dès lors de rétablir, selon les mots de Genissieu lors de la dernière séance de cette tumultueuse assemblée, « union, amitié, concorde entre tous les Français » pour sauver la République.

Par ce travail, à la croisée de la justice et du politique, le Comité de Législation permet à la Convention d'achever son projet politique : clore la Révolution et mettre fin au cycle de revanche et de répression ouvert en thermidor. L'étude de la constitution des dossiers, puis de leur présentation en séance, vient souligner tout le décalage qu'il peut exister entre d'un côté les discours en tribune, la volonté un temps unanime des députés et, de l'autre, la mise en œuvre de ces mêmes volontés dans le secret des comités, puis dans un contexte politique mouvant. Le décret du 8 brumaire, qui devait pourtant protéger les conventionnels de toute mise en cause hâtive, n'est plus en 1795 l'aiguillon suprême, mais bien une référence aléatoire que l'on s'empresse d'oublier en prairial, alors qu'il s'agit d'écarter au plus vite les derniers obstacles à l'élaboration d'une nouvelle Constitution qui doit permettre de «terminer la Révolution ${ }^{54} »$. Ce décalage entre les décrets adoptés et leur application souligne la nécessaire complémentarité entre différentes archives pour mieux appréhender le travail des députés.

\section{NOTES}

1. Félicité DE LAmENNAIS, Le Peuple constituant, cité par Louis MÉNARD, Prologue d'une révolution, février-juin 1848, présentation par Filippo BENFANTE et Maurizio GRIBAUDI, Paris, La Fabrique, 2007, p. 291-292. L'éditorial, trop peu connu, mérite d'être cité in extenso : «Le Peuple constituant a commencé avec la République, il finit avec la République. Car ce que nous voyons, ce n'est pas, certes, la République, ce n'est même rien qui ait un nom : Paris est en état de siège, livré à un pouvoir militaire, livré lui-même à une faction qui en a fait son instrument; les cachots et les forts de Louis-Philippe encombrés de 14000 prisonniers, à la suite d'une affreuse boucherie organisée par des conspirateurs dynastiques, devenus, le lendemain, tout-puissants; des transportations en masse, des proscriptions telles que 95 n'en fournit pas d'exemples; des lois attentatoires au droit de réunion détruit de fait; l'esclavage et la ruine de la presse par l'application monstrueuse de la législation monarchique remise en vigueur, la Garde nationale désarmée en partie, le peuple décimé et refoulé dans sa misère, plus profonde qu'elle ne le fut 
jamais, non, encore une fois, non, certes, ce n'est pas là la République ; mais, autour de sa tombe sanglante, les saturnales de la réaction. »

2. Jaurès, Mathiez, Lefebvre n'ont pas de mot assez durs pour les « réacteurs ", coupables d'avoir tué le rêve révolutionnaire. L'an III, considéré tout au plus comme le marchepied du régime directorial, fut ainsi longtemps un non-lieu de mémoire de l'historiographie révolutionnaire. Voir à ce sujet Pierre SERNA, La République des Girouettes. 1795-1815 et au-delà. Une anomalie politique : la France de l'extrême centre, Seyssel, Champ Vallon, 2005.

3. Marc Antoine BAUDOT, Notes historiques sur la Convention nationale, le Directoire, l'Empire et l'exil des votants, Paris, Imprimerie D. Jouaust, 1893, p. 53.

4. «Thermidor est ce miroir sans magie qui renvoie à chaque révolution naissante la seule image qu'elle ne voudrait pas voir : celle de l'usure et de la décrépitude qui tuent les rêves. » Bronislaw BACZKO, Comment sortir de la Terreur, Thermidor et la Révolution, Paris, Gallimard, 1989, p. 352.

5. Mette Harder a montré la continuation d'une politique épuratoire de part et d'autre du 9 thermidor (27 juillet). Voir Mette HARDER, « “Elle n'a pas même épargné ses membres !" Les épurations de la Convention nationale entre 1793 et 1795 ", Annales historiques de la Révolution française, $\mathrm{n}^{\circ} 381,2015$, p. 77-105.

6. Michel BIARD, La liberté ou la mort. Mourir en député 1792-1795, Paris, Tallandier, 2015, p. 21-41.

7. Howard Brown souligne la continuité du personnel politique de l'an II dans les assemblées de l'an III. Voir Howard G. BROWN, Ending the French Revolution. Violence, Justice, and Repression from the Terror to Napoleon, Charlottesville, University of Virginia Press, 2006, chapitres 6 et 7.

8. Michel BIARD et Hervé LEUWERS (dir.), Visages de la Terreur. L'exception politique de l'an II, Paris, Armand Colin, 2014, p. 4-14.

9. Voir notamment Annie JOURDAN, «Les discours de la terreur à l'époque révolutionnaire. Étude sur une notion ambiguë », French Historical Studies, n 36, 2013, p. 67.

10. Françoise BRUNEL, «L'épuration de la Convention nationale en l'an III », dans Michel Vovelle (dir.), Le Tournant de l'an III. Réaction et Terreur blanche dans la France révolutionnaire, Paris, CTHS, 1997, p. 16.

11. Michel BIARD, La liberté ou la mort... op. cit., p. 83-85.

12. Réimpression de l'ancien Moniteur universel (MU dans la suite), vol. 21, p. 367, séance du le 13 thermidor an II (31 juillet 1794).

13. Françoise BRUNEL, «Les derniers Montagnards et l'unité révolutionnaire ", Annales historiques de la Révolution française, $\mathrm{n}^{\circ} 229,1977$, p. 391.

14. Voir Anne SIMONIN, Le tribunal révolutionnaire de l'an III (août 1794-mai 1795). La justice à l'ordre de tous les jours, Actes du colloque international Justice transitionnelle et Révolution française. L'an III (1794-1795) tenu à Paris les 17 et 18 octobre 2019, Histoire de la Justice, nº 32, 2021, à paraître.

15. Au lendemain du 9 thermidor, la Convention renforce son contrôle sur les représentants en mission et modifie en profondeur la composition des équipes missionnaires. Voir Michel BIARD, «Les pouvoirs des représentants en mission sous la Convention", Annales historiques de la Révolution française, $\mathrm{n}^{\circ} 311,1998$, p. 22-23.

16. Sur ces grands procès et la mise en cause par la Convention des figures "terroristes ", voir, dans les publications récentes, Loris CHAVANETTE, Quatre-vingt-quinze. La Terreur en procès, Paris, CNRS Éditions, 2017. L'auteur y analyse les promesses et des réalisations de la justice pénale mise en place à partir de thermidor, dans le domaine de la répression politique en particulier. Voir aussi Ronen STEINBERG, The Afterlives of the Terror. Facing the Legacies of Mass Violence in Postrevolutionary France, Ithaca, Cornell University Press, 2019, qui explore comment ceux qui ont connu la violence de masse de la Révolution française ont géré cet héritage après thermidor.

17. Michel PERTUE, « La Révolution française et l'abolition de la peine de mort », Annales historiques de la Révolution française, $\mathrm{n}^{\circ} 251,1983$. P. 33.

La Révolution française, 21 | 2021 
18. Germain SICARD, « Conclusion », dans Germain Sicard (dir.), Justice et politique : la Terreur dans la Révolution française, Toulouse, Presses de l'Université de Toulouse, 1997, p. 340.

19. Merlin DE DOUAI, décret présenté par Merlin de Douai au nom des comités de salut public, de sûreté générale et de législation, concernant les formalités de mise en accusation d'un représentant, Archives parlementaires, t. 100, p. 182-183, séance du 8 brumaire an III (29 octobre 1794).

20. $M U$, vol. 24, p. 437, 21 floréal an III (10 mai 1795).

21. Décret du 21 floréal an III (10 mai 1795) portant que dans aucun cas les comités de salut public et de sûreté générale ne seront chargés de l'examen de la conduite des représentants du peuple. Coll. Beaudoin, vol. 61, p. 126.

22. Pierre SERNA, La République des Girouettes, op. cit., p. 366.

23. Ni Mathiez ni Lefebvre n'ont de mots assez durs pour ces hommes de la Plaine, prêts à toutes les compromissions pour obtenir de lucratives responsabilités. Mathiez n'explique l'ascension des légistes que par la «médiocrité générale». Le second donne les noms de Merlin et de Cambacérès pour incarner «l'esprit de la Convention thermidorienne ». Voir Albert MATHIEZ, La réaction thermidorienne [1929], éd. Yannick BOsc et Florence GAUTHIER, Paris, Armand Colin, 2010, p. 34 et p. 125 et George LEFEBVRE, Les thermidoriens 50 Le Directoire, Paris, Armand Colin, 2016 [1937; 1946], p. 26-27.

24. Dans sa thèse pour le diplôme d'archiviste-paléographe soutenue en 2007, Jean-Baptiste Legoff revient sur l'analyse des dénonciations et la constitution progressive des dossiers par le comité : voir Jean-Baptiste LEGOFF, «Représentants face au peuple. Les dénonciations à l'encontre des conventionnels pendant la Terreur et la Réaction thermidorienne ", thèse pour le diplôme d'archiviste-paléographe, École nationale des chartes, 2007.

25. Voir Archives nationales (AN dans la suite), D/III/343-359 et BB/30/30.

26. AN, D/III/343-344.

27. Jacques-Régis DE CAMBACÉRÈs, Mémoires inédits. Éclaircissements publiés par Cambacérès sur les principaux événements de sa vie politique. La Révolution et le Consulat, éd. Laurence CHATEL DE BRANCION, Paris, Perrin, 1999, p. 301.

28. Sergio LuZzATTO, L'automne de la Révolution. Luttes et cultures politiques dans la France thermidorienne, Paris, Honoré Champion, 2001, p. 63.

29. $M U$, vol. 24, p. 600, séance du 10 prairial an III (29 mai 1795).

30. Jacques-Claude Dugué d'Assé, député de l'Orne, compte parmi les représentants réintégrés le 18 frimaire an III (8 décembre 1794).

31. Durand-Maillane ne manque d'ailleurs pas de se « couvrir ", en rappelant qu'il " répugnait à son cœur de se charger de ce rapport ", mais aussi que, depuis longtemps, il souffrait « des torts que certains ne nos collègues faisaient par leur conduite à la représentation nationale ».

32. $M U$, vol. 24, p. 603, séance du 10 prairial an III (29 mai 1795).

33. Ibid.

34. Dugué d'Assé accuse ainsi Mallarmé « d'avoir fait périr des innocents », volé des croix d'or et d'argent, mis en réquisition tout ce qui lui plaisait pour sa table ou encore de «n'avoir jamais rien payé ").

35. $M U$, vol. 24, p. 1038, séance du 18 prairial an III (13 septembre 1795).

36. $M U$, vol. 24, p. 603, séance du 15 germinal an III (4 avril 1795).

37. $M U$, vol. 24, p. 1045, séance du 18 prairial an III (13 septembre 1795).

38. Formule utilisée au sujet de Robespierre. Voir Emmanuel DE LAS CASES, Mémorial de SainteHélène, ou Journal où se trouve consigné, jour par jour, ce qu'a dit et fait Napoléon durant dix-huit mois, Paris, Imprimerie Le Normant, 1823, t. I, p. 424.

39. $M U$, vol. 24, p. 120, séance du 15 germinal an III (4 avril 1795).

40. MU, vol. 25, p. 1301, séance du 25 thermidor an III (13 septembre 1795). 
41. $M U$, vol. 25, p. 1303, séance du 25 thermidor an III (11 septembre 1795).

42. AN, D/III/352. On retrouve dans les archives du Comité des Lettres des « veuves des victimes de Lefiot ".

43. $M U$, vol. 25, p. 1303, séance du 25 thermidor an III (11 septembre 1795).

44. AN, D/III/343.

45. AN, D/III/343, dossier de Jean-Baptiste Massieu.

46. $M U$, vol. 25, p. 1316, séance du 27 thermidor an III (13 septembre 1795).

47. Ibid., p. 1317 .

48. Ce rapport ne sera jamais présenté.

49. Sur ces mémoires, voir Lise ANDRIES, « Récits de survie : les mémoires d'autodéfense pendant l'an II et l'an III ", dans Jean-Claude BONNET (dir.), La carmagnole des Muses. L'homme de lettres et l'artiste dans la Révolution, Paris, Armand Colin, 1988, p. 261-275.

50. AN, D/III/352.

51. AN, D/III/346.

52. Françoise BRUNEL, «Les derniers Montagnards et l'unité révolutionnaire ", op. cit., p. 385-404.

53. Bronislaw ВАСZКо, «Briser la guillotine. Une amnistie thermidorienne», Crime, Histoire \& Sociétés, vol. 8, n² 2, 2004, p. 5-31.

54. Michel TROPER, Terminer la Révolution. La Constitution de 1795, Paris, Fayard, 2006.

\section{RÉSUMÉS}

Alors que la Convention s'interroge sur l'encombrant héritage de l'an II, c'est vers le Comité de Législation que convergent les dénonciations à l'encontre des conventionnels mis en cause à partir de l'automne 1794. La tâche est complexe pour les membres de ce comité qui, en brumaire, se voient chargés de mettre en œuvre une longue procédure pour éviter toute mise en cause infondée. Pourtant, alors que le climat politique se dégrade, cette procédure exposée dans les Archives parlementaires semble de plus en plus négligée. Les archives du comité comme les rapports qu'il propose en séance éclairent davantage l'existence une justice ciblée, qui doit permettre de clore la Révolution en écartant les députés considérés comme dangereux. Ce décalage entre décrets adoptés et leur application parfois aléatoire dans les comités montre bien la nécessaire complémentarité des archives dans l'étude de la période révolutionnaire.

While the Convention evaluates the troublesome heritage from Year II, it is towards the Legislative Committee that, starting in the autumn of 1794, are converging the denunciations against the Convention members allegedly involved. The work is cut out for the Committee members who, in Brumaire, were designated to implement a long procedure to prevent unfounded accusations. And yet, as the political climate deteriorates, this procedure, detailed in the Parliamentary Archives, seems to be increasingly ignored. The Committee's archives, as well as the reports it submits during the plenary sessions of the Convention, give more information about the existence of a targeted justice, one which would make it possible to close the Revolution by removing those deputies deemed dangerous. The discrepancies between the adopted decrees and their sometimes-random implementation within the various committees clearly illustrate the necessary role of the archives in studying the revolutionary era. 
INDEX

Mots-clés : Comité de Législation, Convention thermidorienne, Montagne, dénonciations, an III Keywords : Legislative Committee, Thermidorian Convention, the Mountain, denunciations, year III

\section{AUTEUR}

JEAN D'ANDLAU

IRHIS

Université Lille 3 